\title{
Probing Anomalous Quartic Interactions at the LHC with proton tagging
}

\author{
Cristian Baldenegro ${ }^{1, \star}$ \\ ${ }^{1}$ University of Kansas
}

\begin{abstract}
One of the main goals of the Large Hadron Collider is to find signatures of physics Beyond the Standard Model of particle physics. One way to do this is by studying with high precision the interactions of the Standard Model. In this talk, we address the discovery potential of New Physics in the exclusive channel $p p \rightarrow p X p$ which relies on the general purpose detectors at the Large Hadron Collider and their respective forward proton detector stations, located at about $\sim 210 \mathrm{~m}$ w.r.t. the interaction point. These reactions are highly sensitive to quartic electroweak gauge interactions. As a proof of concept, we discuss the exclusive diphoton production at high diphoton invariant mass. We quote sensitivities on the anomalous $\gamma \gamma \gamma \gamma$ coupling for an integrated luminosity of $300 \mathrm{fb}^{1}$ at the center-of-mass energy of $14 \mathrm{TeV}$. We also discuss the discovery potential of $3 \gamma Z$ anomalous quartic gauge coupling by measuring the $p p \rightarrow p(\gamma \gamma \rightarrow Z \gamma) p$ reaction.
\end{abstract}

\section{Introduction}

One of the primary goals of the Large Hadron Collider (LHC) is the search of signatures of Beyond the Standard Model (BSM) physics. Despite the numerous efforts to find BSM physics at the LHC, none have been successful. Nonetheless, they have ruled out many extension models and have set stronger bounds for their parameter space [1-7]. While these searches are very powerful in specific cases, they end up being highly model-dependent. It is absolutely necessary to exhaust every possibility of New Physics signatures with the best sensitivities accessible at the LHC.

Anomalous quartic gauge interactions between the photons, $W^{ \pm}$, and $Z^{0}$ bosons can be probed with very high precision at the LHC by measuring central exclusive reactions $p p \rightarrow p X p$, where $X=\gamma \gamma, Z \gamma, Z Z, \ell \bar{\ell}$, among others. The system $X$ is reconstructed in the central detector, like ATLAS or CMS, while the outgoing protons, which remain intact after the exchange of quanta carrying the quantum numbers of the vacuum, can be reconstructed with dedicated forward proton detectors at about \pm 210 meters from the nominal interaction point (See Fig. 1). The CMS-TOTEM Precision Proton Spectrometer (CT-PPS) and the ATLAS Forward Physics (AFP) experiments are equipped and operational for the standard high luminosity fills at the LHC to study exclusive and semi-exclusive reactions. The full reconstruction of the final state gives three main advantages: i) due to the kinematic constraint, the number of background events stemming from the simultaneous secondary interactions at the LHC, the pile-up events, is negligible; ii) the production of a massive system $X$ is dominated by photon-exchange events and; iii) systematic uncertainties related to proton dissociation are absent in these reactions, since protons remain intact.

^e-mail: cbaldenegro@ku.edu 


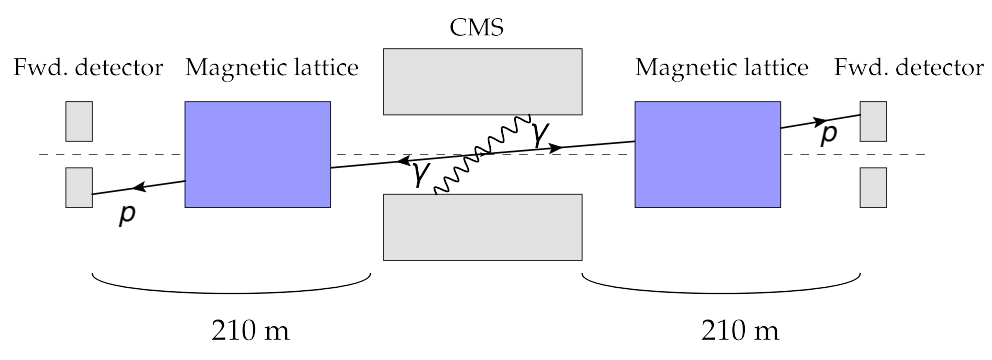

Figure 1. CMS-TOTEM Precision Proton Spectrometer schematic diagram. The $p p \rightarrow p \gamma \gamma p$ reaction is displayed as an example. The diphoton is measured in CMS and the outgoing intact protons with small fractional momentum loss are measured with the forward detectors at $\sim 210 \mathrm{~m}$ w.r.t. the nominal interaction point of CMS. The intact protons are transported through the accelerator's magnetic lattice (in blue), which acts as an effective spectrometer on the intact protons. The final state is reconstructed completely. Distances in this diagram are not to scale.

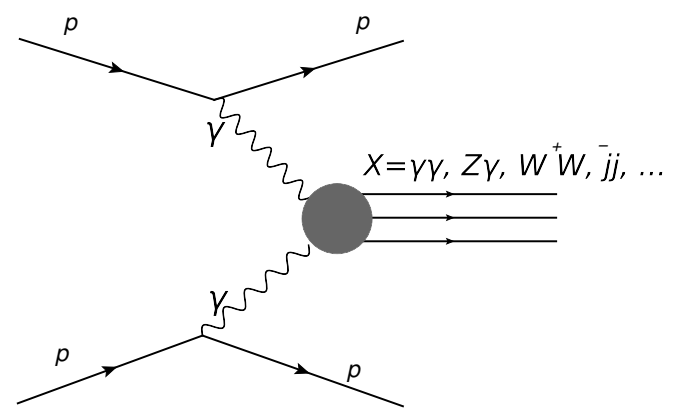

Figure 2. Schematic diagram of $p p \rightarrow p(\gamma \gamma \rightarrow X) p$ reaction. The photon-produced system $X$ is measured in the central detector, the outgoing intact protons are tagged using very forward detectors.

Our focus is on photon-induced processes in $p p$ collisions at high invariant masses, as in Fig. 2. These processes are the most sensitive to the anomalous quartic couplings between the SM vector gauge bosons. Pure gauge interactions can appear due to loops of new real or virtual charged particles. This happens, for instance, in composite Higgs models which require the existence of new charged particles of spin $\frac{1}{2}$ and 1 [8]. New particles with exotic electric charges can also appear in warped extra-dimension models with custodial symmetry [9]. Pure gauge interactions can also be induced via the exchange a resonant neutral state of spin 0 or 2 . A prime example would be the KaluzaKlein [11, 12] graviton.

The CT-PPS experiment has published the first physics results for an integrated luminosity of 10 $\mathrm{fb}^{-1}$ by measuring the semi-elastic production of $\mu^{+} \mu^{-}$pairs using the proton tag method [10]. This first measurement proves that the chain of central and forward detection at the standard high luminosity fills works: from trigger, calibration, alignment and reconstruction has the potential of providing interesting physics results, necessary for future New Physics searches. The AFP experiment started taking data in the Summer of 2017, being an independent experiment probing the same reactions as in CT-PPS at high luminosity fills. ${ }^{1}$

\footnotetext{
${ }^{1}$ Private communication with members of the ATLAS collaboration.
} 

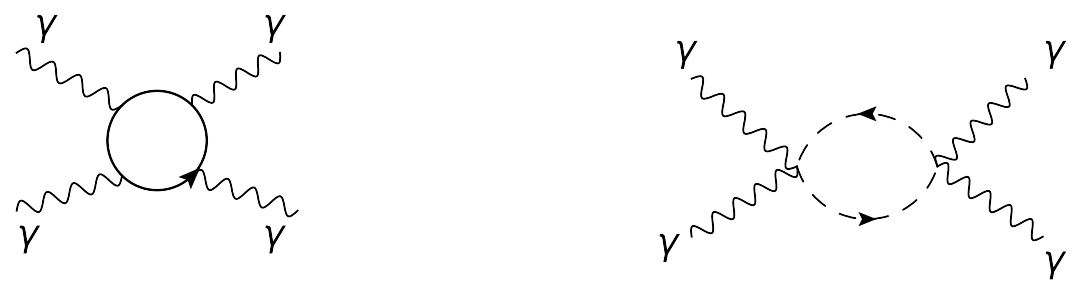

Figure 3. Typical diagrams illustrating electrically charged particles contributing to light-by-light scattering at the lowest order in perturbation theory. (a) The box contribution contains a loop that can involve the SM fermions and $W$-bosons. Any electrically charged BSM particle contributes to this process. (b) Bubble contribution from virtual charged scalars. Only BSM particles contribute to this diagram, since there are no scalars in QED. $t$ channel and $u$-channel diagrams are not shown here.

\section{Light-by-light scattering $(\gamma \gamma \rightarrow \gamma \gamma)$}

Light-by-light scattering $(\gamma \gamma \rightarrow \gamma \gamma)$ was first predicted to exist in QED in 1933 [13]. It was first shown that the leading-order contribution to this process was finite in Ref. [14], but it was not until 2017 that it was first directly observed. Indeed, the first direct evidence of light-by-light scattering was found by the ATLAS Collaboration in $\mathrm{Pb}-\mathrm{Pb}$ collisions [15], setting an important precedent in photon physics at the LHC. ${ }^{2}$ The diphoton mass in this measurement ranges from 10 to $25 \mathrm{GeV}$. Complementary to the ATLAS $\mathrm{Pb}-\mathrm{Pb}$ measurement, the pp process will probe diphoton masses from $\sim 300 \mathrm{GeV}$ to $2 \mathrm{TeV}$ with high transverse momenta photons and with proton tagging. As explained below, this gives sensitivity to regions where the anomalous interaction effects are more important. The Muon g-2 experiment [17] is also sensitive to the four photon coupling, as it modifies directly the QED vertex of the muon. While the precision in this experiment is already exceptional, and the measurement is expected to improve even more at Fermilab, the sensitivity to BSM particle properties in CT-PPS or AFP is expected to be superior [18].

Any new electrically charged particles would contribute to the scattering of light-by-light $(\gamma \gamma \rightarrow$ $\gamma \gamma$ ) via loop diagrams. Light-by-light scattering amplitudes are fully characterized by the mass, spin and electric charge of the particle in the loop [18]. As a result, the search for charged particles can be performed in a fully model-independent way.

As described in [18], using kinematic information from tagged protons can constrain background events by roughly two orders of magnitude. In Fig. 4, we show the leading SM processes which result in two photons and two intact protons in the final state [19-22]. Fig. 2 corresponds to the exclusive QCD diphoton production via colorless two-gluon exchange, while Fig. 2 shows the photon exchange. In Fig 2 we see that quark, lepton and $W^{ \pm}$loops are considered in order to calculate the correct SM cross section for diphoton production. The two-gluon exchange process is dominant at low invariant masses whereas the photon induced ones dominate at higher diphoton masses [23]. This leads to a strong conclusion: if one observes two photons with high invariant mass in the central detector and two intact protons in the forward detectors -and the kinematics are properly balanced between the forward and central detectors- we have high confidence this is due to a photon-induced process.

\footnotetext{
${ }^{2}$ Already limits have been set on the non-linear Born-Infeld extension of QED [16]. This imposed a lower bound on the mass of $\sim 11 \mathrm{TeV}$ of the corresponding magnetic monopole, the strongest to this date.
} 
In the assumption of a new physics mass scale $\Lambda$ heavier than the experimentally accessible energy $E$, all new physics manifestations can be described using an effective Lagrangian valid for $\Lambda \gg E$. Among these operators, the pure photon dimension-eight operators

$$
\mathcal{L}_{4 \gamma}=\zeta_{1}^{4 \gamma} F_{\mu \nu} F^{\mu \nu} F_{\rho \sigma} F^{\rho \sigma}+\zeta_{2}^{4 \gamma} F_{\mu \nu} F^{\nu \rho} F_{\rho \lambda} F^{\lambda \mu}
$$

can induce the $\gamma \gamma \gamma \gamma$ interaction. The unpolarized differential cross section induced by the EFT Lagrangian reads,

$$
\frac{\mathrm{d} \sigma_{\gamma \gamma \rightarrow \gamma \gamma}}{\mathrm{d} \Omega}=\frac{1}{16 \pi^{2} s}\left(s^{2}+t^{2}+s t\right)^{2}\left[48 \zeta_{1}^{2}+40 \zeta_{1} \zeta_{2}+11 \zeta_{2}^{2}\right]
$$

Imposing unitarity on the $S$-wave from the EFT amplitudes, we find the bound

$$
\zeta_{1}, \zeta_{2}<\left(10^{-12}-10^{-11}\right) \mathrm{GeV}^{-4}
$$

In Fig. 2, we see the diphoton invariant mass distribution for signal and backgrounds. As mentioned previously, the signal appears at high diphoton mass. We also see that the dominant background, by several orders of magnitude, at high invariant mass is the $\gamma \gamma+$ pile-up contribution, which is due to the overlap of non-diffractive diphoton production and the presence of two intact protons due to the secondary interactions, commonly known as pile-up interactions. This background can be largely suppressed by applying the rapidity and mass matching criteria (see Fig. 7) between the forward proton detectors information and the central detector information. ${ }^{3}$. The signal selection efficiency exceeds $70 \%$.
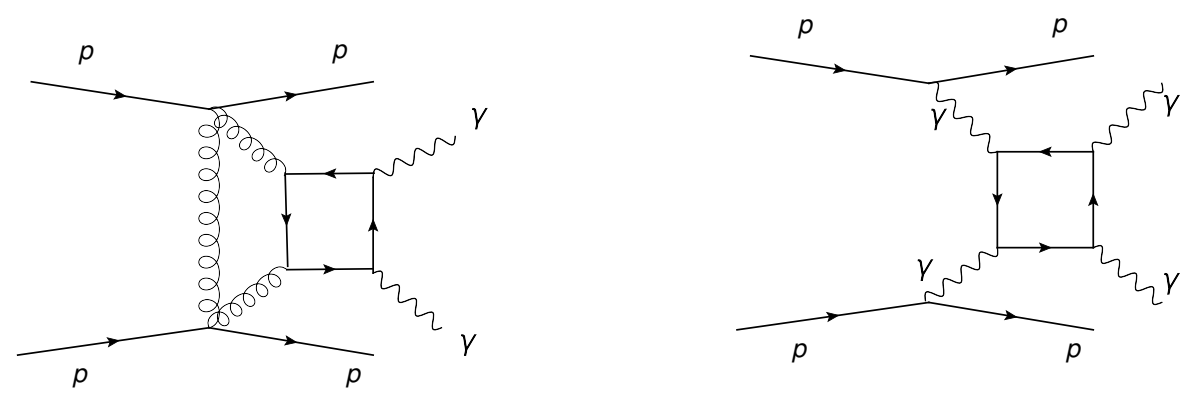

Figure 4. Exclusive $p p \rightarrow p \gamma \gamma p$ processes in the SM at the lowest order in perturbation theory. (a) Two-gluon exchange with a box of virtual charged particles leading to a $\gamma \gamma$ final state, and (b) the well-known light-by-light scattering process with two leading intact protons.

\section{$3 \gamma \gamma \gamma Z$ coupling}

The $\gamma \gamma \gamma Z$ interaction is induced at one-loop level in the SM via loops of fermions and $W^{ \pm}$bosons. Loops of heavy particles charged under $\mathrm{SU}(2)_{L} \times \mathrm{U}(1)_{Y}$ contribute to the $\gamma \gamma \gamma Z$ couplings. These loop contributions only depend on the mass and quantum numbers of the particle in the loop. This anomalous interaction results in the $\gamma \gamma \rightarrow \gamma Z$ scattering and generates the rare SM decay $Z \rightarrow \gamma \gamma \gamma$.

\footnotetext{
${ }^{3}$ The fractional momentum loss of the intact protons $\xi$ is measured by the proton taggers. The diphoton mass can be reconstructed with them via $m_{\gamma \gamma}=\sqrt{\xi_{1} \xi_{2} s}$ (often called missing diproton mass, since it comes from the intact protons) and the diphoton rapidity with $y_{\gamma \gamma}=1 / 2 \log \left(\xi_{1} / \xi_{2}\right)$. This is valid for every central exclusive reaction.
} 


\begin{tabular}{|c||c|c||c|c|c|c|}
\hline Cut / Process & $\begin{array}{c}\text { Signal } \\
\text { (full) }\end{array}$ & $\begin{array}{c}\text { Signal } \\
\text { with (without) } \\
\text { f.f (EFT) }\end{array}$ & Excl. & DPE & $\begin{array}{c}\text { DY, } \\
\text { di-jet } \\
\text { pile up }\end{array}$ & $\begin{array}{c}\gamma \gamma \\
+ \text { pile up }\end{array}$ \\
\hline \hline $\begin{array}{c}{\left[0.015<\xi_{1,2}<0.15,\right.} \\
\left.p_{\mathrm{T} 1,(2)}>200,(100) \mathrm{GeV}\right]\end{array}$ & 65 & $18(187)$ & 0.13 & 0.2 & 1.6 & 2968 \\
$m_{\gamma \gamma}>600 \mathrm{GeV}$ & 64 & $17(186)$ & 0.10 & 0 & 0.2 & 1023 \\
{$\left[p_{\mathrm{T} 2} / p_{\mathrm{T} 1}>0.95\right.$,} & 64 & $17(186)$ & 0.10 & 0 & 0 & 80.2 \\
$|\Delta \phi|>\pi-0.01]$ & 61 & $16(175)$ & 0.09 & 0 & 0 & 2.8 \\
$\sqrt{\xi_{1} \xi_{2} s}=m_{\gamma \gamma} \pm 3 \%$ & 60 & $12(169)$ & 0.09 & 0 & 0 & 0 \\
$\left|y_{\gamma \gamma}-y_{p p}\right|<0.03$ & & & & & \\
\hline
\end{tabular}

Figure 5. Number of signal events for a given coupling value and background events after various selections for an integrated luminosity of $300 \mathrm{fb}^{-1}$ and $\mu=50$ at $\sqrt{s}=14 \mathrm{TeV}$. At least one converted photon is required. Excl. stands for exclusive backgrounds and DPE for double pomeron exchange backgrounds.
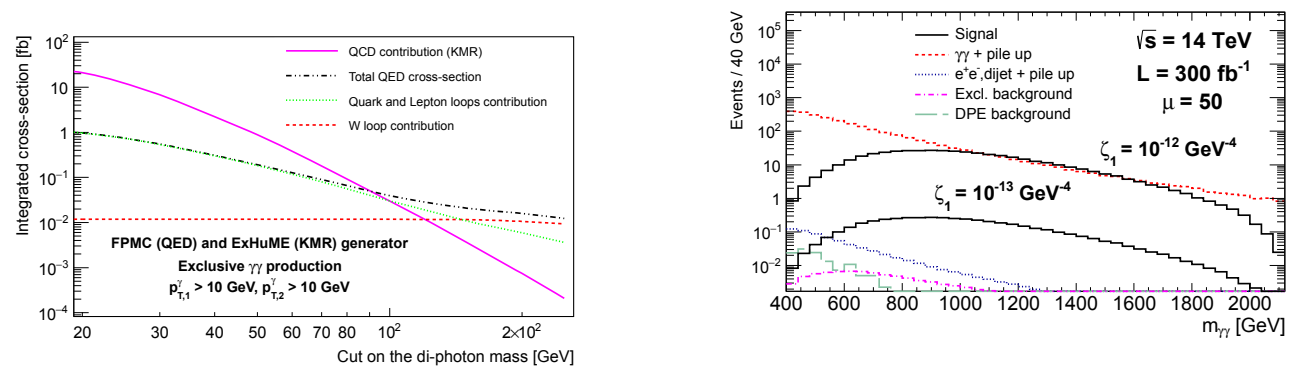

Figure 6. (a) SM exclusive diphoton cross-section as a function of the diphoton inv. mass cut. Considered events involve two intact protons in the forward proton detectors and photons with $p_{\mathrm{T}}>10$. The QCD contribution (two-gluon exchange in magenta) dominates at low masses, while QED (diphoton production dashed lines) dominates at higher masses. The QED production contains two pieces: lepton/fermion loops (dotted line) and $W$ boson loops (dashed-dotted line). (b) Diphoton invariant mass distribution for the signal $\left(\zeta_{1}=10^{-12}, 10^{-13} \mathrm{GeV}^{-4}\right)$ and for the backgrounds (dominated by $\gamma \gamma$ with protons from pile-up), requesting two protons in the forward detectors and two photons of $p_{\mathrm{T}}>50 \mathrm{GeV}$.

If a $\gamma \gamma \gamma \gamma$ signal is observed, the $p p \rightarrow p(\gamma \gamma \rightarrow \gamma Z) p$ channel would provide a clear test whether or not the underlying exchanged particle is universally coupled to the gauge kinetic terms.

In the presence of New Physics with a mass scale $\Lambda$ heavier than the experimentally accessible energy $E$, all New Physics manifestations can be described using an effective Lagrangian valid for $\Lambda \gg E$. In this low-energy EFT, the $\gamma \gamma \gamma Z$ interactions are described by two dimension-eight $\gamma \gamma \gamma Z$ operators

$$
\mathcal{L}_{\gamma \gamma \gamma Z}=\zeta O^{\gamma Z}+\tilde{\zeta} \tilde{O}^{\gamma Z}=\zeta F^{\mu \nu} F_{\mu \nu} F^{\rho \sigma} Z_{\rho \sigma}+\tilde{\zeta} F^{\mu \nu} \tilde{F}_{\mu \nu} F^{\rho \sigma} \tilde{Z}_{\rho \sigma},
$$

with $\tilde{F}^{\mu \nu}=\frac{1}{2} \epsilon^{\mu \nu \rho \sigma} F_{\rho \sigma}$. The $O_{2}^{\gamma Z}=F^{\mu v} F_{\nu \rho} F^{\rho \sigma} Z_{\sigma \mu}$ operator can sometimes be encountered in the literature. It is related to the above basis via the identity $4 O_{2}^{\gamma Z}=2 O^{\gamma Z}+\tilde{O}^{\gamma Z}$. The $O^{\gamma Z}, \tilde{O}^{\gamma Z}$ provides a somewhat clearer mapping onto the properties of the underlying physics.

We find the unpolarized differential cross section to be

$$
\frac{\mathrm{d} \sigma_{\gamma \gamma \rightarrow \gamma Z}^{\mathrm{NP}}}{\mathrm{d} \Omega}=\frac{\beta}{16 \pi^{2} s}\left[\left(3 \zeta^{2}+3 \tilde{\zeta}^{2}-2 \zeta \tilde{\zeta}\right)(s t+t u+u s)^{2}-4\left(\zeta^{2}+\tilde{\zeta}^{2}-\zeta \tilde{\zeta}\right)^{2} m_{Z}^{2} s t u\right]
$$



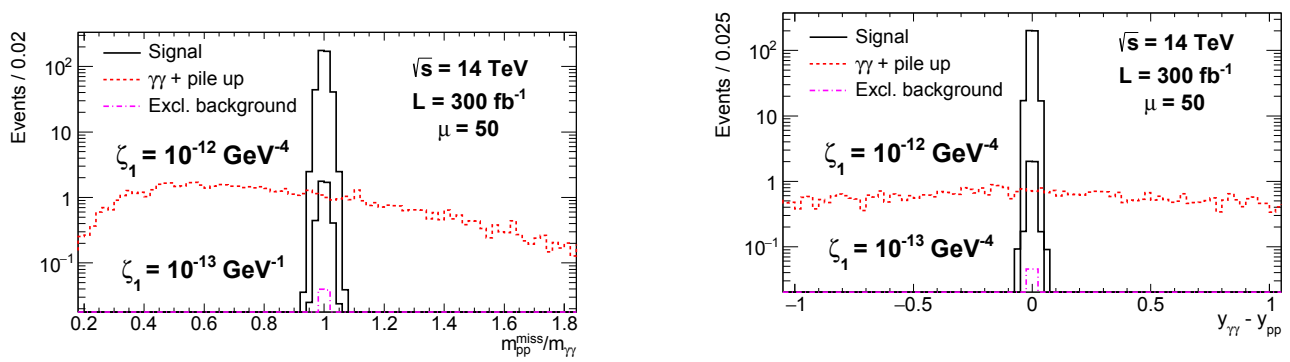

Figure 7. Missing diproton mass $m_{p p}=\sqrt{\xi_{1} \xi_{2} s}$ to central mass ratio distribution (Top) and rapidity difference distribution (Bottom) in the $p \gamma \gamma p$ channel for the signal and background within the fractional momentum loss acceptance $0.015<\xi_{1,2}<$ 0.15 considering two different coupling values after applying the required cuts on $p_{\mathrm{T}}$, invariant mass $m_{\gamma \gamma}, p_{T}$ ratios and angle separation. The integrated luminosity is $300 \mathrm{fb}^{-1}$ and the average pile-up is $\mu=50$. Exclusive processes peak at unity and zero respectively.

where $s, t$, and $u$ are the usual Mandelstam variables and $\beta=1-m_{Z}^{2} / s$ for the $\gamma Z$ final state.

By imposing unitarity on the $S$-wave of the EFT amplitudes and neglecting the $Z$ boson mass one finds the conditions

$$
|\zeta+\tilde{\zeta}| s^{2}<4 \pi, \quad|\zeta-\tilde{\zeta}| s^{2}<\frac{12 \pi}{5} .
$$

The discovery potential of this anomalous coupling via the exclusive production $p p \rightarrow p(\gamma \gamma \rightarrow$ $Z \gamma) p$ has been addressed in detail in [24]. The EFT approach is used in the study, and conclusions to scenarios where loops of new particles charged under $\mathrm{SU}(2)_{L} \times \mathrm{U}(1)_{Y}$ or neutral resonance production. Two dimension- 8 operators contribute to the anomalous quartic interaction $3 \gamma Z$ with couplings $\zeta, \tilde{\zeta}$. Just as in the exclusive diphoton production, the only significant background is the overlap of non-diffractive events with $\gamma Z$ final states and the simultaneous intact protons coming from the uncorrelated single diffractive and double diffractive events. This background can be reduced by comparing the forward and central measurements, as seen in Fig. 8 for the $p p \rightarrow p(\gamma \gamma \rightarrow Z \gamma) p \rightarrow p(\gamma \gamma \rightarrow$ Dijet $+\gamma) p$ case.
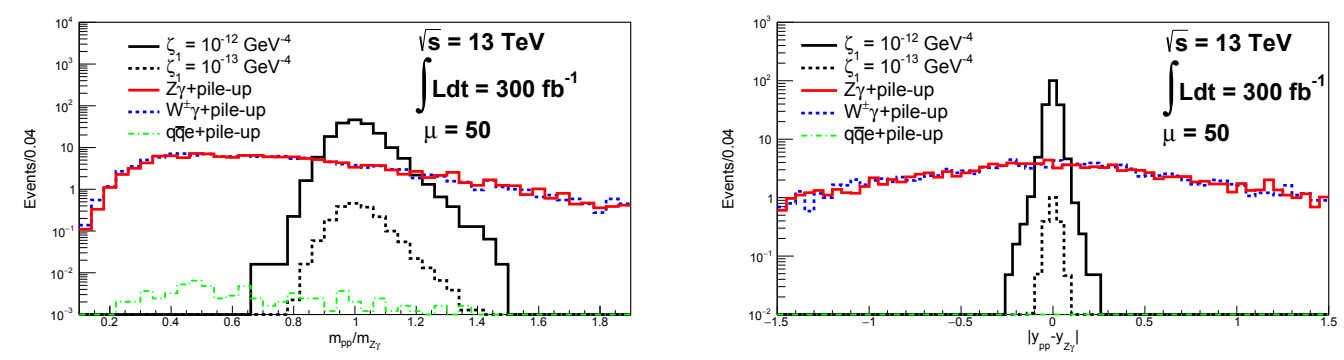

Figure 8. Missing diproton mass $m_{p p}=\sqrt{\xi_{1} \xi_{2} S}$ to central mass ratio distribution (Top) and rapidity difference distribution (Bottom) in the $j j \gamma$ channel for the signal and background within the acceptance $0.015<\xi_{1,2}<0.15$ considering two different coupling values after applying the requirement on $p_{\mathrm{T}}$, invariant mass $m_{Z \gamma}, p_{T}$ ratios and angle separation. The integrated luminosity is $300 \mathrm{fb}^{-1}$ and the average pile-up is $\mu=50$. The signal width is due to a combined effect of the reconstructed jet energy low resolution $(\approx 15 \%)$ and the $\xi_{1,2}$ resolution from the proton detectors. The asymmetry on the $m_{p p} / m_{Z \gamma}$ distribution is due to the resolution on the jet energy. 


\begin{tabular}{|c||c|c||c|c|}
\hline \multicolumn{1}{|c||}{ Coupling $\left(\mathrm{GeV}^{-4}\right)$} & \multicolumn{2}{c||}{$\zeta(\tilde{\zeta}=0)$} & \multicolumn{2}{c|}{$\zeta=\tilde{\zeta}$} \\
\hline Luminosity & \multicolumn{2}{c||}{$300 \mathrm{fb}^{-1}$} & \multicolumn{2}{c|}{$300 \mathrm{fb}^{-1}$} \\
\hline Pile-up $(\mu)$ & \multicolumn{2}{|c|}{50} & \multicolumn{2}{c|}{50} \\
\hline Channels & $5 \sigma$ & $95 \% \mathrm{CL}$ & $5 \sigma$ & $95 \%$ CL \\
\hline$\ell \bar{\ell} \gamma$ & $2.8 \cdot 10^{-13}$ & $1.8 \cdot 10^{-13}$ & $2.5 \cdot 10^{-13}$ & $1.5 \cdot 10^{-13}$ \\
\hline$j j \gamma$ & $2.3 \cdot 10^{-13}$ & $1.5 \cdot 10^{-13}$ & $2 \cdot 10^{-13}$ & $1.3 \cdot 10^{-13}$ \\
\hline$j j \gamma \bigoplus \ell \bar{\ell} \gamma$ & $1.93 \cdot 10^{-13}$ & $1.2 \cdot 10^{-13}$ & $1.7 \cdot 10^{-13}$ & $1 \cdot 10^{-13}$ \\
\hline
\end{tabular}

Table 1. $5 \sigma$ discovery and $95 \% \mathrm{CL}$ exclusion limits on $\zeta, \tilde{\zeta}$. Sensitivities are given for $300 \mathrm{fb}^{-1}$ for $\mu=50$ pile-up interactions. The last row corresponds to the search of $Z \gamma$ production in both channels with their respective selection cuts.

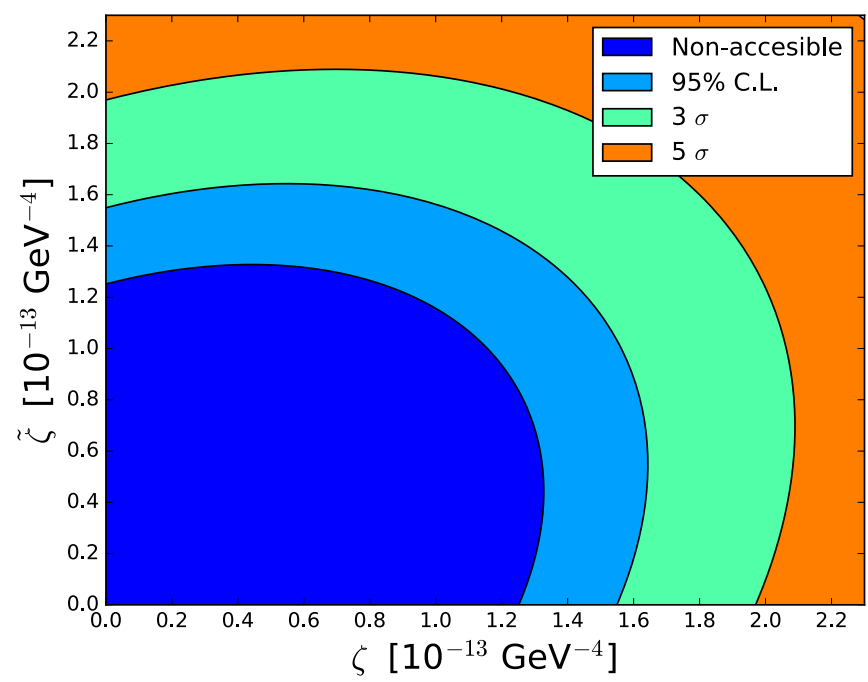

Figure 9. Sensitivity in the $\zeta-\tilde{\zeta}$ plane for $300 \mathrm{fb}^{-1}$ and $\mu=50$. Orange, green and light blue can be probed at 5 $\sigma, 3 \sigma$, and $95 \%$ C.L. using proton tagging at the LHC.

The exclusive channel allows the possibility of studying exclusive $Z \gamma$ production with the $Z$ decaying leptonically or hadronically. The signature $\ell \bar{\ell} \gamma$ is much cleaner, but generates vastly fewer events than $q \bar{q} \gamma$. The sensitivity on the anomalous coupling considering both decay channels at 13 $\mathrm{TeV}$, with $300 \mathrm{fb}^{-1}$ of data, is $1.7 \cdot 10^{-13} \mathrm{GeV}^{-4}$ at $5 \sigma$ and $1 \cdot 10^{-13} \mathrm{GeV}^{-4}$ at $95 \% \mathrm{CL}$.

The strongest bound on the branching ratio $\mathcal{B}(Z \rightarrow \gamma \gamma \gamma)$ was found by the ATLAS Collaboration at $8 \mathrm{TeV}$ [25]: $\mathcal{B}(Z \rightarrow \gamma \gamma \gamma)<2.2 \cdot 10^{-6}$. This bound translates to a limit on the anomalous couplings

$$
\left[\zeta^{2}+\tilde{\zeta}^{2}-\frac{1}{2} \zeta \tilde{\zeta}\right]^{1 / 2}<1.3 \cdot 10^{-9} \mathrm{GeV}^{-4}(95 \% \mathrm{CL})
$$


Imagining that the same search is done at $13 \mathrm{TeV}$ with $300 \mathrm{fb}^{-1}$, we roughly expect this bound to improve by an order of magnitude. This remains far away from the expected sensitivities obtained in the exclusive channel at the same luminosity by roughly three orders of magnitude.

\section{Conclusions}

The exclusive channel provides an excellent test ground to probe quartic electroweak gauge boson interactions with cross-sections at the order of fractions of fb. Search of quartic interactions in the exclusive channel allows to study New Physics in a model-independent. The couplings can be characterized as a function of the mass, spin and charge under $\mathrm{SU}(2)_{L} \times \mathrm{U}(1)_{Y}$ of the new particles contributing via loops in $\gamma \gamma \rightarrow \gamma \gamma$ and $\gamma \gamma \rightarrow \gamma Z$ scattering.

The CT-PPS experiment has published the first results for an integrated luminosity of $10 \mathrm{fb}^{-1}$ for the semi-elastic production of $\mu^{+} \mu^{-}$pairs at the LHC using the proton tagging method. This first measurement proves that the chain of exclusive and forward detection works at the standard high luminosity fills, which goes from trigger, calibration, alignment and reconstruction can provide interesting physics results, necessary for future BSM searches. The ATLAS Forward Physics project started taking data in the Summer of 2017, being an independent experiment probing the same reactions as in CT-PPS.

\section{References}

[1] CMS Collaboration. Search for signatures of extra dimensions in the diphoton mass spectrum at the Large Hadron Collider. Phys. Rev. Lett., 108:111801, 2012.

[2] CMS Collaboration. Search for quark compositeness in dijet angular distributions from pp collisions at $\sqrt{s}=7$ TeV. JHEP, 05:055, 2012.

[3] CMS Collaboration. Search for large extra dimensions in dimuon and dielectron events in pp collisions at $\sqrt{s}=7$ TeV. Phys. Lett., B711:15-34, 2012.

[4] ATLAS Collaboration. Search for pair production of heavy vector-like quarks decaying to highpT W bosons and b quarks in the lepton-plus-jets final state in pp collisions at $\sqrt{s}=13 \mathrm{TeV}$ with the ATLAS detector. 2017.

[5] ATLAS Collaboration. A search for resonances decaying into a Higgs boson and a new particle $\mathrm{X}$ in the XH qqbb final state with the ATLAS detector. 2017.

[6] CMS Collaboration. Search for low mass vector resonances decaying into quark-antiquark pairs in proton-proton collisions at $\sqrt{s}=13 \mathrm{TeV}$. 2017.

[7] CMS Collaboration. Search for dark matter produced with an energetic jet or a hadronically decaying $\mathrm{W}$ or $\mathrm{Z}$ boson at $\sqrt{s}=13 \mathrm{TeV}$. JHEP, 07:014, 2017.

[8] K. Agashe, R. Contino, and A. Pomarol. The Minimal composite Higgs model. Nucl. Phys., B719:165-187, 2005.

[9] K. Agashe, A. Delgado, M.J. May, and R. Sundrum. RS1, custodial isospin and precision tests. JHEP, 08:050, 2003.

[10] CMS and TOTEM collaborations, Evidence for proton-tagged, central semi-exclusive production of high-mass muon pairs at $13 \mathrm{TeV}$ with the CMS-TOTEM Precision Proton Spectrometer, CMS-PAS-PPS-17-001, 2017.

[11] Th. Kaluza. Zum Unitatsproblem in der Physik. Sitzungsber. Preuss. Akad. Wiss. Berlin (Math. Phys.), page 966, 1921.

[12] O. Klein. Quantentheorie und funfdimensionale Relativitatstheorie. Zeitschrift fur Physik, 37(12):895-906, Dec 1926. 
[13] O. Halpern. Scattering Processes Produced by Electrons in Negative Energy States. Phys. Rev., 44:855.2-856, 1933.

[14] H. Euler and B. Kockel. Ueber die Streuung von Licht an Licht nach der Diracschen Theorie. Naturwiss., 23:246, 1935.

[15] ATLAS Collaboration. Measurement of exclusive $\mathrm{W}+\mathrm{W}$ production and search for exclusive Higgs boson production in pp collisions at $\sqrt{s}=8 \mathrm{TeV}$ using the ATLAS detector. Phys. Rev., D94(3):032011, 2016.

[16] J. Ellis, N.E. Mavromatos, and T. You. Light-by-Light Scattering Constraint on Born-Infeld Theory. Phys. Rev. Lett., 118(26):261802, 2017.

[17] G. Venanzoni. The Fermilab Muon g 2 Experiment. PoS, EPS-HEP2015:568, 2015.

[18] S. Fichet, G. von Gersdorff, B. Lenzi, C. Royon, and M. Saimpert. Light-by-light scattering with intact protons at the LHC: from Standard Model to New Physics. JHEP, 02:165, 2015.

[19] E. Chapon, C. Royon, and O. Kepka. Anomalous quartic W +W , ZZ, and trilinear W +W couplings in two-photon processes at high luminosity at the LHC. Phys. Rev., D81:074003, 2010.

[20] O. Kepka and C. Royon. Anomalous W W coupling in photon-induced processes using forward detectors at the LHC. Phys. Rev., D78:073005, 2008.

[21] S. Fichet, G. von Gersdorff, and C. Royon. Measuring the Diphoton Coupling of a $750 \mathrm{GeV}$ Resonance. Phys. Rev. Lett., 116(23):231801, 2016.

[22] S. Fichet, G. von Gersdorff, and C. Royon. Scattering light by light at $750 \mathrm{GeV}$ at the LHC. Phys. Rev., D93(7):075031, 2016.

[23] V. A. Khoze, A. D. Martin, and M. G. Ryskin. Prospects for new physics observations in diffractive processes at the LHC and Tevatron. Eur. Phys. J., C23:311-327, 2002.

[24] C. Baldenegro, S. Fichet, Gero von Gersdorff, and C. Royon. Probing the anomalous Z coupling at the LHC with proton tagging. JHEP, 06:142, 2017.

[25] ATLAS Collaboration. Search for new phenomena in events with at least three photons collected in pp collisions at $\sqrt{s}=8 \mathrm{TeV}$ with the ATLAS detector. Eur. Phys. J., C76(4):210, 2016. 